\title{
A importância da assistência farmacêutica nos problemas relacionados à medicamentos: uma revisão integrativa
}

\author{
The importance of pharmaceutical assistance in drug-related problems: an integrative review
}

La importancia de la atención farmacéutica en los problemas relacionados con las drogas: una revisión integradora

\section{Resumo}

$\mathrm{O}$ acesso aos medicamentos permite a cura e o tratamento de diversas enfermidades, proporcionando qualidade e aumento na expectativa de vida aos usuários. Entretanto a utilização dos medicamentos também podem provocar efeitos indesejáveis e impedir a evolução do tratamento, caracterizando os problemas relacionados à medicamentos (PRMs), que desenvolvem-se durante as fases do processo terapêutico, como a prescrição, dispensação e administração ou resultante do processo nocivo esperado no tratamento. A finalidade desse trabalho, visa demonstrar a importância do profissional farmacêutico na detecção, prevenção e resolução dos problemas resultantes da farmacoterapia. Para embasar a elaboração, foi pesquisado artigos originais, nas bases de dados, SciELO, Pubmed e LILACS. A partir da análise dos estudos, percebe-se que a presença de erros na farmacoterapia é bem frequente, em diversos públicos, comprometendo a segurança do paciente e causando ainda mais dano, além de elevar os custos do sistema, por isso ressalta-se a importância do farmacêutico, atuando de forma preventiva ao aparecimento dos PRMs, fazendo intervenções e reconciliação medicamentosa com o objetivo de reduzir erros, interações, reações adversas e proporcionando otimizar a farmacoterapia.

Palavras-chave: Farmacoterapia; Farmacêutico; PRMs.

\begin{abstract}
O Access to medicines allows for the cure and treatment of various diseases, providing quality and increased life expectancy for users. However, the use of drugs can also cause undesirable effects and prevent the evolution of treatment, characterizing drug-related problems (DRPs), which develop during phases of the therapeutic process, such as prescription, dispensing and administration or resulting from the harmful process expected in the treatment. The purpose of this work is to demonstrate the importance of the pharmacist in detecting, preventing and solving problems resulting from pharmacotherapy. To support the elaboration, original articles were searched, in the databases, SciELO, Pubmed and LILACS. From the analysis of the studies, it is clear that the presence of errors in pharmacotherapy is quite frequent, in various audiences, compromising patient safety and causing even more damage, in addition to raising the costs of the system, so it is important to emphasize the importance of the pharmacist, acting in a preventive way to the emergence of DRPs, making interventions and drug reconciliation in order to reduce errors, interactions, adverse reactions and providing optimize pharmacotherapy.
\end{abstract}

Keywords: Pharmacotherapy; Pharmaceutical; DRPs.

\section{Resumen}

El acceso a los medicamentos permite la cura y el tratamiento de diversas enfermedades, proporcionando calidad y mayor esperanza de vida a los usuarios. Sin embargo, el uso de medicamentos también puede provocar efectos indeseables y prevenir la evolución del tratamiento, caracterizando los problemas relacionados con los medicamentos $(\mathrm{PRM})$, que se desarrollan durante las fases del proceso terapéutico, como la prescripción, dispensación y administración o como resultado del proceso nocivo esperado. en el tratamiento. El propósito de este trabajo es demostrar la importancia del farmacéutico en la detección, prevención y solución de problemas derivados de la farmacoterapia. Para apoyar la elaboración se realizaron búsquedas de artículos originales, en las bases de datos SciELO, Pubmed y LILACS. Del análisis de los estudios se desprende que la presencia de errores en la farmacoterapia es bastante frecuente, en diversos públicos, comprometiendo la seguridad del paciente y provocando aún más daño, además de elevar los costos del sistema, por lo que es importante resaltar la importancia del 
farmacéutico, actuando de forma preventiva ante la aparición de PRMs, realizando intervenciones y conciliaciones de fármacos con el fin de reducir errores, interacciones, reacciones adversas y ofreciendo optimizar la farmacoterapia.

Palabras clave: Farmacoterapia; Farmacéutico; PMRs.

\section{Introdução}

O desenvolvimento dos medicamentos ao longo dos anos representou uma evolução na atenção à saúde, visto que proporcionou o tratamento de diversas patologias, permitindo qualidade e aumento na expectativa de vida aos pacientes, porém o uso dos medicamentos também pode provocar malefícios (Spalla \& Castilho, 2016). Os Problemas relacionados à medicamentos (PRMs) são eventos que se desenvolvem durante o tratamento medicamentoso, podendo estar associados com a necessidade de indicação medicamentosa, eficácia ou segurança do tratamento (Rodrigues et al., 2019).

Os PRMs podem serem resultantes das etapas que envolvem o processo terapêutico, como a prescrição, dispensação e administração, mas também do efeito nocivo que pode ser esperado no tratamento. A ocorrência dos PRMs, prejudica a farmacoterapia e podem causar danos aos pacientes (Nascimento et al., 2020), esse resultado diferente do que é almejado na terapêutica, provoca consultas extras e hospitalizações, o que colabora com o aumento nos custos de saúde (Casper et al., 2019). Os efeitos nocivos ao paciente desenvolvido da terapia, constitui as reações adversas a medicamentos ou erros de medicação (EM), caracterizando os PRMs (Aguiar et al., 2018).

O cenário atual favorece o aumento de reações adversas. Após a declaração pela OMS em 12 de março de 2020, que a doença pelo coronavírus (COVID-19), se caracterizava como uma pandemia, e causando ao longo dos meses vários danos, altas internações e mortes, houve uma utilização em massa de fármacos sem orientação, com o objetivo de prevenir e/ou amenizar esse patógeno (Melo et al., 2021).

As reações adversas aos medicamentos (RAM) são definidas como um evento que causa efeitos indesejáveis, sendo desenvolvido durante a utilização dos medicamentos, mesmo com doses usualmente empregadas (Modesto et al., 2016). Algumas associações de medicamentos inadequadas favorecem o desenvolvimento de reações adversas e interações medicamentosas (Nascimento et al., 2017). Além disso, influenciam o desenvolvimento de RAM: idade, sexo, gênero e morbidades (Modesto et al., 2016)

Os erros de medicação são desencadeados durante o uso dos medicamentos, caracterizando-se como um incidente que pode causar dano no paciente ou influenciar no uso inadequado. Devido a segurança do paciente ser um grande desafio nos últimos anos, a reconciliação medicamentosa contribui com o cuidado contínuo do paciente, sendo uma barreira para prevenir interferências nesse processo (Spalla \& Castilho, 2016).

A partir da Resolução 585 de 2013 do Conselho Federal de Farmácia (CFF), tem a expansão da atuação do profissional farmacêutico, regulamentando as atribuições clínicas. Essa ampliação dos serviços clínicos surge em virtude da alta morbimortalidade decorrente de doenças e agravos não transmissíveis e à farmacoterapia (de farmácia, 2013). A intervenção farmacêutica promove uma terapia segura e eficiente, a partir da identificação, resolução e prevenção dos problemas relacionados aos medicamentos (PRMs) (Viana et al., 2017; Aguiar et al., 2018).

Os serviços de revisão de medicamentos, permite que o farmacêutico otimize a farmacoterapia e obtenha melhora da qualidade de vida do paciente, pois a partir de uma avaliação dos medicamentos prescritos e conforme o histórico de medicamentos do paciente, além das informações fornecidas permite fazer intervenções personalizadas para resolver aqueles problemas identificados na prescrição (Makovec et al., 2021). Dessa forma, são relatados em vários estudos os benefícios resultantes da farmácia clínica, seja na atenção primária ou ambiente hospitalar, pois proporciona o uso racional e adequado dos medicamentos, por meio da reconciliação ou revisões medicamentosas (Pfister et al., 2017). 
A partir de todo contexto apresentado, o objetivo desse trabalho visa demonstrar a importância que o profissional farmacêutico pode proporcionar na detecção, prevenção e resolução dos PRMs, permitindo otimizar o tratamento medicamentoso e minimizar a chance de efeitos indesejáveis.

\section{Metodologia}

Trata-se de uma revisão integrativa da literatura, realizada a partir de pesquisas nas bases de dados eletrônicas: SciELO (Scientific Electronic Library Online), PubMed e LILACS (Literatura Latino-Americana e do Caribe em Ciências da Saúde), para a busca foi utilizado descritores em Ciências da Saúde (DeCS): Assistência farmacêutica, erros de medicação, segurança do paciente e medicamentos. Além disso, utilizou-se os termos: "Problemas relacionados a medicamentos", no idioma inglês. Também foi utilizado materiais, do Conselho Federal de Farmácia e da Agência Nacional de Vigilância Sanitária (ANVISA). Para a realização desse trabalho foi seguido as seis fases para elaboração da revisão integrativa, que consiste em: elaboração da pergunta de pesquisa, busca dos artigos na literatura, coleta de dados, análise dos estudos, discussão dos resultados e apresentação da revisão (Souza et al.; 2010).

Foi selecionado artigos originais, completos e disponíveis gratuitamente em qualquer idioma. Os estudos utilizados para construir a revisão, deverá apresentar um recorte temporal dos últimos cinco anos- 2016 à 2021. Para uma elegibilidade inicial, serão considerados os títulos de cada registro para verificar compatibilidade com o tema abordado. Após analisar os títulos dos estudos e os resumos, o texto será analisado para extrair as informações mais relevantes acerca do tema pesquisado. Todo material utilizado foi devidamente citado, respeitando os direitos autorais e propriedade intelectual.

Tabela 1: Estratégias de busca, quantidade de artigos encontrados nas bases de dados e utilizados.

\begin{tabular}{|c|c|c|c|c|}
\hline Bases de dados & $\begin{array}{l}\text { Estratégias de busca: } \\
\text { Decs/Palavras chaves }\end{array}$ & Artigos encontrados & $\begin{array}{c}\text { Filtros: recorte } \\
\text { temporal, tipo de } \\
\text { estudo e } \\
\text { disponibilidade. }\end{array}$ & Artigos utilizados \\
\hline SciELO & $\begin{array}{c}\text { "medicamentos AND } \\
\text { segurança AND } \\
\text { paciente" }\end{array}$ & 107 & 42 & 10 \\
\hline LILACS & $\begin{array}{c}\text { "Assistência } \\
\text { farmacêutica AND erros } \\
\text { de medicação" }\end{array}$ & 50 & 18 & 4 \\
\hline Pubmed & "drug related problems" & 40.841 & 25 & 8 \\
\hline
\end{tabular}

Fonte: Elaboração própria (2021).

\section{Resultados e Discussão}

Para a elaboração desse trabalho, foram utilizadas 25 referências, incluindo em sua maioria estudos encontrados nas bases de dados mencionadas anteriormente. No quadro abaixo contém características gerais dos principais artigos utilizados para o desenvolvimento desse tópico. 
Quadro 1: Descrição dos artigos, conforme o título, autores e ano, objetivos e periódico publicado.

\begin{tabular}{|c|c|c|c|}
\hline Título & Autores e ano & Objetivo (s) & Periódico \\
\hline $\begin{array}{c}\text { Problemas relacionados a } \\
\text { medicamentos em neonatos } \\
\text { cardiopatas sob terapia intensiva }\end{array}$ & $\begin{array}{l}\text { Nascimento, A. R. F. D., } \\
\text { Leopoldino, R. W. D., Santos, M. } \\
\text { E. T. D., Costa, T. X. D., \& } \\
\text { Martins, R. R. (2020). }\end{array}$ & $\begin{array}{l}\text { Estabelecer a frequência e as } \\
\text { características dos PRMs em pacientes } \\
\text { neonatos cardiopatas de uma unidade de } \\
\text { terapia intensiva (UTIN). }\end{array}$ & $\begin{array}{l}\text { Revista Paulista de } \\
\text { Pediatria }\end{array}$ \\
\hline $\begin{array}{l}\text { Problemas relacionados a drogas e } \\
\text { análises de medicamentos entre } \\
\text { idosos com demência }\end{array}$ & $\begin{array}{l}\text { Pfister, B., Jonsson, J., \& } \\
\text { Gustafsson, M. (2017). }\end{array}$ & $\begin{array}{l}\text { Avaliar a ocorrência e o caráter dos } \\
\text { problemas relacionados aos } \\
\text { medicamentos em idosos com demência } \\
\text { ou deficiência cognitiva. }\end{array}$ & $\begin{array}{l}\text { BMC Pharmacology and } \\
\text { Toxicology }\end{array}$ \\
\hline $\begin{array}{l}\text { Análise do serviço de farmácia } \\
\text { clínica em um hospital } \\
\text { universitário }\end{array}$ & $\begin{array}{c}\text { Cruz, L. T., do Nascimento } \\
\text { Batista, P., \& Meurer, I. R. (2019). }\end{array}$ & $\begin{array}{c}\text { Detectar os PRMs e as intervenções } \\
\text { farmacêuticas, realizadas na Farmácia de } \\
\text { um Hospital Universitário da } \\
\text { Universidade Federal de Juiz de Fora } \\
\text { (HU-UFJF), além de apresentar as taxas } \\
\text { de aceitação dessas intervenções pela } \\
\text { equipe de saúde. }\end{array}$ & HU REVISTA \\
\hline $\begin{array}{l}\text { Relação entre o uso seguro de } \\
\text { medicamentos e serviços de } \\
\text { farmácia clínica em Unidades de } \\
\text { Cuidados Intensivos Pediátricos }\end{array}$ & $\begin{array}{l}\text { Okumura, L. M., da Silva, D. M., } \\
\text { \& Comarella, L. (2016). }\end{array}$ & $\begin{array}{l}\text { Discutir a atuação da implantação de um } \\
\text { serviço de farmácia clínica na Unidade de } \\
\text { Cuidados Intensivos Pediátricos (UCIP) } \\
\text { brasileira, direcionada à beira do leito. }\end{array}$ & $\begin{array}{l}\text { Revista Paulista de } \\
\text { Pediatria }\end{array}$ \\
\hline $\begin{array}{l}\text { Segurança do paciente e o valor da } \\
\text { intervenção em um hospital } \\
\text { oncológico }\end{array}$ & $\begin{array}{c}\text { Aguiar, K. D. S., Santos, J. M. D., } \\
\text { Cambrussi, M. C., Picolotto, S., \& } \\
\text { Carneiro, M. B. (2018). }\end{array}$ & $\begin{array}{l}\text { Apresentar o impacto financeiro obtido a } \\
\text { partir do papel do farmacêutico envolvido } \\
\text { na identificação e na prevenção de erros } \\
\text { das prescriçôes do tipo antineoplásicos. }\end{array}$ & Einstein (São Paulo) \\
\hline $\begin{array}{c}\text { Reações adversas a medicamentos } \\
\text { em pacientes com COVID-19 no } \\
\text { Brasil: análise das notificações } \\
\text { espontâneas do sistema de } \\
\text { farmacovigilância brasileiro }\end{array}$ & $\begin{array}{l}\text { Melo, J. R. R., Duarte, E. C., } \\
\text { Moraes, M. V. D., Fleck, K., } \\
\text { Silva, A. S. D. N., \& Arrais, P. S. } \\
\text { D. (2021) }\end{array}$ & $\begin{array}{c}\text { Avaliar as RAMs nos pacientes } \\
\text { diagnosticados com COVID- } 19, \\
\text { identificados no período, } 1^{\circ} \text { de março à } 15 \\
\text { de agosto de } 2020, \text { no Brasil, bem como } \\
\text { os fatores relacionados ao surgimento de } \\
\text { reações graves. }\end{array}$ & Cadernos de Saúde Pública \\
\hline $\begin{array}{l}\text { A dispensação de medicamentos } \\
\text { como oportunidade de } \\
\text { aconselhamento ao paciente e } \\
\text { abordagem de problemas } \\
\text { relacionados a medicamentos. }\end{array}$ & $\begin{array}{l}\text { Ferreira, T. X. A. M., Prudente, L. } \\
\text { R., Dewulf, N. D. L. S., Provin, } \\
\text { M. P., Mastroianni, P. D. C., } \\
\text { Silveira, E. A. D., \& Amaral, R. } \\
\text { G. (2016). }\end{array}$ & $\begin{array}{c}\text { Descrever e avaliar processo de } \\
\text { dispensação de medicamentos criado e } \\
\text { inserido em uma farmácia comunitária, } \\
\text { localizado numa universidade pública de } \\
\text { Goiás, Brasil. }\end{array}$ & $\begin{array}{c}\text { Revista Brasileira de } \\
\text { Ciências Farmacêuticas }\end{array}$ \\
\hline $\begin{array}{l}\text { Intervenções do farmacêutico } \\
\text { clínico em uma unidade de } \\
\text { cuidados intermediários com foco } \\
\text { no paciente idoso. }\end{array}$ & $\begin{array}{l}\text { Viana, S. D. S. C., Arantes, T., \& } \\
\text { Ribeiro, S. C. D. C. (2017). }\end{array}$ & $\begin{array}{l}\text { Discutir a atuação do farmacêutico clínico } \\
\text { no atendimento hospitalar de pacientes } \\
\text { idosos em situação crítica. }\end{array}$ & Einstein (São Paulo) \\
\hline $\begin{array}{c}\text { Gerenciamento de problemas } \\
\text { relacionados à farmacoterapia na } \\
\text { síndrome coronariana aguda: papel } \\
\text { do farmacêutico clínico na unidade } \\
\text { de reabilitação cardíaca }\end{array}$ & $\begin{array}{l}\text { Casper, E. A., El Wakeel, L. M., } \\
\text { Saleh, M. A., \& El-Hamamsy, M. } \\
\text { H. (2019). }\end{array}$ & $\begin{array}{l}\text { Avaliar o impacto dos efeitos que os } \\
\text { serviços desenvolvidos por farmacêuticos } \\
\text { clínicos têm no desfecho de pacientes com } \\
\text { síndrome coronariana aguda. }\end{array}$ & $\begin{array}{l}\text { Basic \& clinical } \\
\text { pharmacology \& } \\
\text { toxicology }\end{array}$ \\
\hline $\begin{array}{c}\text { Maior adesão ao serviço de } \\
\text { Revisão de Uso de Medicamentos } \\
\text { na Eslovênia: um ensaio clínico } \\
\text { randomizado }\end{array}$ & $\begin{array}{c}\text { Makovec, U. N., Locatelli, I., \& } \\
\text { Kos, M. (2021). }\end{array}$ & $\begin{array}{l}\text { Avaliar os benefícios desenvolvidos pelo } \\
\text { serviço de Revisão de Uso de } \\
\text { Medicamentos na Eslovênia, focando no } \\
\text { impacto da adesão à medicação. }\end{array}$ & $\begin{array}{l}\text { BMC health services } \\
\text { research }\end{array}$ \\
\hline $\begin{array}{c}\text { Orientação farmacêutica na alta } \\
\text { hospitalar de pacientes transplantados: } \\
\text { estratégia para a segurança do } \\
\text { paciente }\end{array}$ & $\begin{array}{l}\text { Lima, L. F., Martins, B. C. C., } \\
\text { Oliveira, F. R. P. D., Cavalcante, } \\
\text { R. M. D. A., Magalhães, V. P., } \\
\text { Firmino, P. Y. M., ... \& Néri, E. D. } \\
\text { R. (2016). }\end{array}$ & $\begin{array}{c}\text { Descrever e analisar a atuação da } \\
\text { orientação farmacêutica na alta hospitalar } \\
\text { de pacientes transplantados. }\end{array}$ & Einstein (São Paulo) \\
\hline $\begin{array}{c}\text { Serviços prestados por } \\
\text { farmacêuticos clínicos em crianças } \\
\text { graves com sobrecarga de ferro } \\
\text { beta-talassemia: uma nova visão } \\
\text { sobre o atendimento ao paciente. }\end{array}$ & $\begin{array}{c}\text { Bahnasawy, S. M., El Wakeel, L. } \\
\text { M., Beblawy, N. E., \& } \\
\text { El-Hamamsy, M. (2017). }\end{array}$ & $\begin{array}{c}\text { Avaliar o impacto do serviço realizado } \\
\text { por farmacêuticos clínicos em crianças } \\
\text { com beta-talassemia associado a } \\
\text { sobrecarga de ferro. }\end{array}$ & $\begin{array}{l}\text { Basic \& clinical } \\
\text { pharmacology \& } \\
\text { toxicology }\end{array}$ \\
\hline
\end{tabular}




\subsection{Principais Problemas Relacionados à Medicamentos}

A utilização concomitantemente de cinco ou mais medicamentos, caracterizada como polifarmácia (Marquito et al., 2020) é uma prática comum na clínica, porém se combinados de forma inadequada, contribuem com problemas relacionados à medicamentos (PRMs) (Nascimento et al., 2017), pois a quantidade de medicamentos pode interferir na qualidade da prescrição e adesão, principalmente em idosos (Geurts et al., 2016). Os PRMs, caracterizam-se como um problema de saúde envolvendo a terapia medicamentosa, que pode influenciar nos resultados esperados da terapêutica e na qualidade de vida do paciente (Marquito et al., 2020). Reações adversas, interações, uso inapropriado dos medicamentos, prescrições inadequadas e falta de medicação, são alguns impasses que podem surgir na farmacoterapia (Gustafsson et al., 2017).

Um grupo muito propenso ao aparecimento dos PRMs, são os neonatos, devido apresentarem imaturidade fisiológica, alterações de peso dificultando a posologia das doses e o uso off label dos medicamentos, ocasionada pela falta de medicamentos próprios para essa população. Em um estudo transversal, feito em uma Unidade de Terapia Intensiva Neonatal (UTIN), identificou-se em 122 neonatos cardiopatas, 390 PRMs, tendo destaque, efetividade do tratamento (49,0\%) e reação adversa (46,7\%). A principal causa relacionada, foi o processo de uso do medicamento e erro na dose, tendo a vancomicina, meropenem e furosemida, os fármacos mais relacionados com os PRMs (Nascimento et al., 2020).

A utilização de medicamentos aprovados para adultos em crianças e adolescentes, favorecem uma maior suscetibilidade desses grupos ao aparecimento de problemas na terapêutica. Em uma Unidade de Terapia Intensiva Pediátrica em Campo largo, no Brasil foram detectados 141 PRMs em 35 pacientes, sendo a incompatibilidade das soluções intravenosas, doses elevadas, farmacoterapia duplicada, dose incompatível com o clearance renal, tempo de infusão errado e dose baixa, as principais detectadas pelo serviço de farmácia clínica (Okumura et al., 2016).

Outro grupo bastante vulnerável de ocorrer falhas no tratamento medicamentoso são os idosos, tendo altas possibilidades de ocorrer: reações adversas, interações e uso potencialmente inadequado dos fármacos (Pfister et al., 2017), pois o envelhecimento traz alterações fisiológicas que podem comprometer os processos de farmacocinética e farmacodinâmica, por isso o monitoramento é necessário (Viana et al., 2017). Entre os 310 PRMs identificados, em pacientes com 65 anos ou mais que apresentavam demência, ou deficiência cognitiva, foram classificados em sete subgrupos: doses adequadas resultando em RAM, dosagem muito alta, dosagem muito baixa, drogas ineficazes/inapropriadas, necessidade de terapia medicamentosa adicional, terapia medicamentosa desnecessária, abandono, interações, erros de transição e necessidade de monitoramento (Pfister et al., 2017).

\subsection{Erros de medicação}

Nos serviços de saúde, os erros de medicação tem grande prevalência, devido falhas na prestação do cuidado, por isso a segurança do paciente é um tema que vem sendo bastante discutido na atualidade (Cruz et al., 2019), compreendendo que segurança é a capacidade de reduzir o risco de dano desnecessário ao indivíduo associado ao cuidado em saúde (Nascimento et al., 2017). Os erros de medicamentos são incidentes resultantes de vários fatores, mas os principais relatados na literatura estão envolvidos com os processos de prescrição, dispensação e administração, permitindo diferença entre o medicamento prescrito e o medicamento administrado (Palma Arellano et al., 2018).

O erro de prescrição é um dos processos terapêuticos que possui grande chance de provocar consequências danosas ao paciente, seja devido sua elaboração manual ou relacionado a informações incompletas ou incorretas (Nascimento et al., 2020; Palma Arellano et al.,2018). Em um estudo realizado por Aguiar et al. (2018) em um hospital de ensino, especializado em oncologia no sul do Brasil, das 6.014 prescrições analisadas, 274 apresentaram algum erro relacionado aos medicamentos, o PRM de maior relevância foi relacionado as prescrições devido falta de informações, o que permite o surgimento de dúvidas e falhas pelos profissionais envolvidos. 
A dispensação é um processo importante, pois nela é fornecido informações a cerca do tratamento prescrito, permitindo que o paciente compreenda os medicamentos que utilizará, pois a falta de orientação ao paciente pode causar má adesão, efeitos indesejáveis, falha terapêutica, efeitos adversos e interferir na evolução do paciente. Através das etapas que envolvem a dispensação o farmacêutico poderá identificar PRM, fazer intervenções e contribuir com o uso adequado pelo paciente e sucesso na terapia (Ferreira et al., 2016).

Durante a administração também é possível ocorrer interferências no processo, por isso a presença de instruções na prescrição sobre essa etapa, como horário da administração do medicamento, poderá evitar interações com outros fármacos ou com alimento, impedindo alteração no efeito e na absorção respectivamente (Marquito et al., 2020). Fármacos da classe dos antibióticos como ciprofloxacina e doxiciclina, bem como levotiroxina e bifosfonatos, possuem redução na sua absorção se administrados simultaneamente por via oral com medicamentos compostos por cátions di e trivalentes (Pfister et al., 2017).

Os erros de medicação é o que mais afeta a segurança do paciente e possui alta possibilidade de ser evitado. Apesar de contribuir com o aumento da morbidade hospitalar, não necessariamente será desenvolvido nesse ambiente, porém a maioria são provenientes nesses locais, podendo ser desencadeado devido falhas na comunicação pelos profissionais das equipes multidisciplinares, perda de informações essenciais para o cuidado durante seu trajeto no hospital, informações incompletas ou ausentes contribuindo com interferências na terapêutica (Santos et al., 2019). Dessa forma, práticas voltadas ao uso racional dos medicamentos tem alta possibilidade de impedir o acontecimento desses erros, além disso a presença do farmacêutico no serviço permite uma maior identificação (Palma Arellano et al., 2018).

\subsection{Automedicação da população e os impactos na saúde}

$\mathrm{O}$ uso de medicamentos de forma autônoma favorece a prática irracional, sendo um problema de saúde pública bastante presente na sociedade, assim o farmacêutico é um profissional com papel em disseminar a utilização dos medicamentos de maneira correta, contribuindo com a minimização de riscos e custos relacionados à farmacoterapia (Aguiar et al., 2018). Por isso a dispensação é uma função desse profissional que visa orientar o paciente, para promover o uso adequado dos medicamentos e alcançar os resultados almejados da terapia (Ferreira et al., 2016).

Visando frear a disseminação do coronavírus, a utilização de fármacos como hidroxicloroquina, cloroquina e azitromicina, foram bastantes buscados devido apresentarem in vitro atividade antiviral, além disso, foi recomendado em alguns países o uso terapêutico contra o vírus, porém ainda é experimental, pois os estudos não garantem segurança e eficácia, contra o coronavírus. Devido essa utilização, esses fármacos estão associados a manifestações de reações adversas, relatados riscos de cardiotoxicidade, prolongamento do intervalo QT, permitindo o aparecimento de arritmias cardíacas e fibrilação ventricular, mas também de diarreias, prurido e elevação das transaminases, favorecendo problemas hepáticos (Melo et al., 2021).

A Agência Nacional de Vigilância Sanitária (ANVISA), divulgou o comunicado 3/2021, para alertar sobre os riscos de eventos adversos provenientes do uso indiscriminado de medicamentos, sem orientação profissional e sobre a importância de notificar esses eventos no VigiMed, mesmo se for apenas uma suspeita, para evitar possíveis reações graves e óbitos (Anvisa, 2021). Melo et al., (2021), incluíram em um estudo, 402 relatórios de segurança de casos individuais, provenientes do centro nacional de monitorização de medicamentos (CNMM) da Anvisa, para analisar a presença de RAM em pacientes com COVID-19. Foram detectadas 631 RAMs em 402 pacientes com uma média de 1,6 por paciente, os mais envolvidos com suspeitas ou interação, foram hidroxicloroquina, azitromicina, cloroquina e a ceftriaxona. Os farmacêuticos, foram os que mais notificaram casos suspeitos de RAM ao sistema de farmacovigilância. 


\subsection{Importância do Farmacêutico na redução dos PRMS}

O papel do farmacêutico em avaliar a prescrição, pode identificar fatores envolvidos no desenvolvimento dos PRMs, atuando de forma preventiva à possíveis danos decorrentes da terapia e favorecendo segurança. Uma etapa importante é a avaliação da farmacoterapia, pois ela permite verificar as indicações, segurança e eficácia dos medicamentos prescritos e se o paciente adere à terapia (Geurts et al., 2016). Em uma unidade de farmácia hospitalar de um hospital universitário, foi analisado 18.795 prescrições, referentes aos meses de abril de 2018 à outubro de 2018, sendo detectados 2.834 PRMs. As intervenções farmacêuticas tiveram $70 \%$ de aceitação, mostrando a credibilidade da farmácia clínica em minimizar a incidência dessas falhas (Cruz et al., 2019).

Um estudo em uma farmácia comunitária de uma universidade pública, com 104 pacientes mostrou a importância do processo de dispensação. Foi possível identificar e corrigir os PRMs, em mais de $60 \%$ das intervenções realizadas, sendo as principais detectadas relacionadas com a falta de conhecimentos do paciente sobre seu tratamento medicamentoso. A identificação e solução das intervenções realizadas pelos farmacêuticos contribuiu com o maior conhecimento dos medicamentos, evitando má adesão, resultados indesejáveis, falhas da terapêutica e intoxicações de saúde (Ferreira et al., 2016)

Foi demostrado que em pacientes com síndrome coronariana aguda, o acompanhamento de 3 meses do farmacêutico clínico, o seu papel de contribuir com um melhor gerenciamento e resultados dos pacientes colaborou com mudanças significativas no grupo teste. Foram feitas 138 intervenções no grupo teste, indicado como controlar os efeitos colaterais dos fármacos $(44,2 \%)$, adição de medicamentos (20,2\%), suspensão de medicamentos $(8 \%)$, substituição de medicamentos (14\%) e ajuste de dose (14\%), tendo uma aceitação de $96,2 \%$. As classes mais relacionadas com os PRMs, em ambos os grupos, teste e controle, foram: Beta- bloqueadores (21,9\%), estatinas (17,3\%) e IECA/ARBs (15,3\%) (Casper et al., 2019).

Um serviço de farmácia clínica realizado com pacientes pediátricos com beta-talassemia, teve resultados significativos no grupo intervenção, após 6 meses de acompanhamento. No início do estudo foram detectados 64 PRMs, sendo problema na adesão o mais identificado, após as intervenções durante o estudo a quantidade total de PRMs foi reduzida para 4, tendo $93,75 \%$ de aceitação, confirmando o impacto positivo no atendimento ao paciente que esse profissional tem em otimizar o tratamento do paciente (Bahnasawy et al., 2017).

\subsection{Benefícios para o paciente da Intervenção Farmacêutica}

A intervenção farmacêutica, consiste em um processo planejado e documentado, buscando uma otimização da farmacoterapia, associada com a promoção, proteção e a recuperação da saúde, permitindo prevenir doenças e/ou problemas de saúde (de farmácia, 2013). Assim, o profissional farmacêutico na equipe, tem a responsabilidade de ajustar as doses dos medicamentos prescritos, permitir adesão ao tratamento, monitorar exames laboratoriais, educar o paciente, além de proporcionar alternativas medicamentosas, tudo isso visando obter os melhores resultados no manejo clínico (Casper et al., 2019).

Solicitação de inclusão do medicamento (n: 39), solicitação de ajuste da dose do medicamento (n: 6), adequação ao processo de dispensação do fármaco (n: 6) e solicitação de exames (n: 4), foram as principais intervenções farmacêuticas (IF) realizadas durante o momento da alta de 74 pacientes transplantados renais e/ou hepáticos. Como foram identificadas 59 PRMs, foram feitas $59 \mathrm{IF}$, sendo todas aceitas, das quais cerca de 98,3\% delas, o médico foi o profissional contatado. Com as intervenções realizadas, pode-se evitar o desfecho clínico que poderia ocorrer caso o PRM persistisse, reduzindo a possibilidade do surgimento de eventos adversos e garantindo a segurança do paciente (Lima et al., 2016).

Almejando uma melhor adesão ao tratamento a partir da identificação de fatores particulares do indivíduo para intervir de forma personalizada, um grupo de farmacêuticos comunitários certificados para realizar a Revisão de uso de medicamentos, proporcionou melhorar a adesão em pacientes com baixa adesão à medicação, evitando resultados de saúde 
ruins (Makovec et al., 2021). Por isso, uma revisão regular prestada pela assistência farmacêutica, através de um acordo com o paciente, busca otimizar o impacto dos medicamentos utilizados, minimizar a chance ocorrer PRMs e reduzir o desperdício associado (Geurts et al., 2016).

\subsection{Reconciliação medicamentosa- Farmacêutico}

A reconciliação medicamentosa, visa prevenir erros resultantes do processo de medicação, a partir análise da prescrição já que o paciente está propenso a erros durante o processo de assistência a saúde (Santos et al., 2019). A análise de prescrição pode ser feita a partir de uma avaliação da lista de medicamentos prescritos ou através de uma revisão clínica dos medicamentos, a partir da análise dos dados clínicos do paciente, associado com uma anamnese, sendo possível identificar e reduzir problemas relacionados a medicamentos (Verdoorn et al., 2019). Os serviços de reconciliação ou de revisões de medicamentos na farmácia clínica, permite uma avaliação individual do tratamento prescrito ao paciente, buscando otimizar a terapêutica (Pfister et al., 2017).

O papel da equipe multidisciplinar é tão importante, assim todos envolvidos na terapia medicamentosa, da prescrição até a administração precisa cooperar com a prevenção de erros (Santos et al., 2019). O farmacêutico clínico, realiza a reconciliação medicamentosa, a partir da revisão e avaliação das prescrições médicas atuais e correlacionando com às prescrições anteriores e a clínica do paciente, permitindo identificar divergências que possam comprometer a evolução do indivíduo (Rodrigues et al., 2019).

Além de atuar no período de internação esse profissional também atua na alta hospitalar, sendo uma etapa que requer cuidados, pois tem uma alta vulnerabilidade de ocorrer problemas na farmacoterapia. Assim o farmacêutico com a equipe, é responsável por orientar ao paciente como dará continuidade ao tratamento, fazer conciliação medicamentosa, identificação de problemas na prescrição, permitindo alcançar as metas terapêuticas do problema de saúde atual e prevenir possíveis eventos que possam comprometer a terapia (Lima et al., 2016).

$\mathrm{O}$ aumento da expectativa de vida, favorece o crescimento de idosos na população, e isso possibilita a prevalência de Doenças Crônicas Não Transmissíveis (DCNT) e polifarmácia. O acompanhamento de 62 idosos pelo farmacêutico em uma unidade de cuidados intermediários, possibilitou 212 intervenções em 386 prescrições avaliadas. Das intervenções, 38,7\% se referia a indicações farmacoterapêuticas, mas também foi realizado intervenções para o uso racional de medicamentos. Os medicamentos potencialmente inapropriados (MPI), foram detectados 196 vezes nas prescrições. As intervenções farmacêuticas foram significativamente aceitas, potencializando seu papel para o cuidado ao paciente (Viana et al., 2017).

\section{Considerações Finais}

O desenvolvimento dos medicamentos ao longo dos anos proporcionou uma facilidade no acesso de boa parte da população, proporcionando melhora de sinais e sintomas e cura de várias enfermidades, porém esse arsenal terapêutico disponível também aumenta a susceptibilidade a danos provocados pelo uso errôneo dos medicamentos. Os problemas relacionados a medicamentos, são bastantes frequentes na sociedade, e provocam efeitos indesejáveis, retardando o processo de melhora. São vários fatores que estão associados como o surgimento dessa problemática, que gera prejuízos ao paciente e também aos sistemas de saúde.

Embora a profissão farmacêutica seja milenar, a compreensão do seu papel ainda é pouco conhecido na sociedade. A atuação desse profissional, não se limita a parte gerencial, pois também atua diretamente com o paciente, como demonstrado em vários estudos, contribuindo com a qualidade de vida. Ademais, o seu papel foi fundamental para otimizar a terapêutica medicamentosa, obter efeitos mais satisfatórios e garantir segurança a partir da redução dos riscos ao paciente. 
Dessa forma, sugere-se o desenvolvimento de mais trabalhos direcionados a atuação dos farmacêuticos na redução dos PRMs, a partir de estudos nos serviços de saúde onde tem o papel desse profissional bem definido na farmácia clínica e a elaboração de estudos bibliográficos utilizando outras bases de dados, ambos serão importantes para nortear pesquisadores, acadêmicos, profissionais e população interessada.

\section{Referências}

Aguiar, K. D. S., Santos, J. M. D., Cambrussi, M. C., Picolotto, S., \& Carneiro, M. B. (2018). Patient safety and the value of pharmaceutical intervention in a cancer hospital. Einstein (Sao Paulo), 16.

Anvisa, Agência Nacional de Vigilância Sanitária. (2021). Anvisa alerta para riscos do uso indiscriminado de medicamentos. https://www.gov.br/anvisa/ptbr/assuntos/noticias-anvisa/2021/anvisa-alerta-para-riscos-do-uso-indiscriminado-de-medicamentos

Bahnasawy, S. M., El Wakeel, L. M., Beblawy, N. E., \& El-Hamamsy, M. (2017). Clinical pharmacist-provided services In iron-overloaded beta-thalassaemia major children: a new insight Into patient care. Basic \& clinical pharmacology \& toxicology, 120(4), 354-359.

Casper, E. A., El Wakeel, L. M., Saleh, M. A., \& El-Hamamsy, M. H. (2019). Management of pharmacotherapy-related problems in acute coronary syndrome: Role of clinical pharmacist in cardiac rehabilitation unit. Basic \& clinical pharmacology \& toxicology, 125(1), 44-53.

Cruz, L. T., do Nascimento Batista, P., \& Meurer, I. R. (2019). Análise do serviço de farmácia clínica em um hospital universitário. HU Revista, 45(4), 408414.

de Farmácia, C. F. (2013). Resolução n 585, de 29 de agosto de 2013. Regulamenta as atribuições clínicas do farmacêutico e dá outras providências. Brasília: Diário Oficial da União, 2013. management recod in hospital. Rev Bras Farm Hosp Serv Saude, 10(4), 0370.

Ferreira, T. X. A. M., Prudente, L. R., Dewulf, N. D. L. S., Provin, M. P., Mastroianni, P. D. C., Silveira, E. A. D., \& Amaral, R. G. (2016). Medication dispensing as an opportunity for patient counseling and approach to drug-related problems. Brazilian Journal of Pharmaceutical Sciences, $52,151-162$.

Geurts, M. M., Stewart, R. E., Brouwers, J. R., de Graeff, P. A., \& de Gier, J. J. (2016). Implications of a clinical medication review and a pharmaceutical care plan of polypharmacy patients with a cardiovascular disorder. International journal of clinical pharmacy, 38(4), 808-815.

Gustafsson, M., Sjölander, M., Pfister, B., Jonsson, J., Schneede, J., \& Lövheim, H. (2017). Pharmacist participation in hospital ward teams and hospital readmission rates among people with dementia: a randomized controlled trial. European journal of clinical pharmacology, 73(7), 827-835.

Lima, L. F., Martins, B. C. C., Oliveira, F. R. P. D., Cavalcante, R. M. D. A., Magalhães, V. P., Firmino, P. Y. M., \& Néri, E. D. R. (2016). Pharmaceutical orientation at hospital discharge of transplant patients: strategy for patient safety. Einstein (São Paulo), 14, $359-365$.

Makovec, U. N., Locatelli, I., \& Kos, M. (2021). Improved adherence with Medicines Use Review service in Slovenia: a randomized controlled trial. BMC health services research, 21(1), 1-12.

Marquito, A. B., Pinheiro, H. S., \& Paula, R. B. D. (2020). Adaptação transcultural do instrumento PAIR: Pharmacotherapy Assessment in Chronic Renal Disease para aplicação no Brasil. Ciência \& Saúde Coletiva, 25, 4021-4032.

Melo, J. R. R., Duarte, E. C., Moraes, M. V. D., Fleck, K., Silva, A. S. D. N., \& Arrais, P. S. D. (2021). Reações adversas a medicamentos em pacientes com COVID-19 no Brasil: análise das notificações espontâneas do sistema de farmacovigilância brasileiro. Cadernos de Saúde Pública, 37, e00245820.

Modesto, A. C. F., Ferreira, T. X. A. M., Provin, M. P., Amaral, R. G., \& Lima, D. M. (2016). Reações adversas a medicamentos e farmacovigilância: conhecimentos e condutas de profissionais de saúde de um hospital da rede sentinela. Revista Brasileira de Educação Médica, 40, 401-410.

Nascimento, A. R. F. D., Leopoldino, R. W. D., Santos, M. E. T. D., Costa, T. X. D., \& Martins, R. R. (2020). Problemas relacionados a medicamentos em neonatos cardiopatas sob terapia intensiva. Revista Paulista de Pediatria, 38.

Nascimento, R. C. R. M. D., Álvares, J., Guerra Junior, A. A., Gomes, I. C., Silveira, M. R., Costa, E. A., \& Acurcio, F. D. A. (2017). Polifarmácia: uma realidade na atenção primária do Sistema Único de Saúde. Revista de Saúde Publica, 51, 19s.

Okumura, L. M., da Silva, D. M., \& Comarella, L. (2016). Relação entre o uso seguro de medicamentos e serviços de farmácia clínica em Unidades de Cuidados Intensivos Pediátricos. Revista Paulista de Pediatria, 34, 397-402.

Palma Arellano, H., Lomelí Terán, J. M., Morales Chávez, G., \& Poblano Morales, M. N. (2018). Impacto do seguimento farmacoterapêutico para identificar erros de medicação e diminuir eventos adversos em pacientes na Terapia Intensiva do Hospital H+ Querétaro. Medicina crítica (Colegio Mexicano de Medicina Crítica), 32(2), 61-65.

Pfister, B., Jonsson, J., \& Gustafsson, M. (2017). Drug-related problems and medication reviews among old people with dementia. BMC Pharmacology and Toxicology, 18(1), 1-11

Rodrigues, J. P. V., Marques, F. A., Gonçalves, A. M. R. F., Campos, M. S. D. A., Reis, T. M. D., Morelo, M. R. S., \& Pereira, L. R. L. (2019). Analysis of clinical pharmacist interventions in the neurology unit of a Brazilian tertiary teaching hospital. PLoS One, 14(1), e0210779.

Santos, C. O. D., Lazaretto, F. Z., Lima, L. H., Azambuja, M. S., \& Millão, L. F. (2019). Reconciliação de medicamentos: processo de implantação em um complexo hospitalar com a utilização de sistema eletrônico. Saúde em Debate, 43, 368-377. 
Research, Society and Development, v. 10, n. 15, e167101522662, 2021

(CC BY 4.0) | ISSN 2525-3409 | DOI: http://dx.doi.org/10.33448/rsd-v10i15.22662

Spalla, L. D. R., \& Castilho, S. R. D. (2016). Medication reconciliation as a strategy for preventing medication errors. Brazilian Journal of Pharmaceutical Sciences, 52, 143-150.

Souza, M. T. D., Silva, M. D. D., \& Carvalho, R. D. (2010). Revisão integrativa: o que é e como fazer. Einstein (São Paulo), 8, 102-106.

Verdoorn, S., Kwint, H. F., Blom, J. W., Gussekloo, J., \& Bouvy, M. L. (2019). Effects of a clinical medication review focused on personal goals, quality of life, and health problems in older persons with polypharmacy: a randomised controlled trial (DREAMeR-study). PLoS medicine, 16(5), e1002798.

Viana, S. D. S. C., Arantes, T., \& Ribeiro, S. C. D. C. (2017). Interventions of the clinical pharmacist in an Intermediate Care Unit for elderly patients. Einstein (São Paulo), 15, 283-288. 